\title{
Operation Control for Tidal Stream Turbines
}

\author{
Meizheng Li ${ }^{1, a}$,Zhaohang Liu ${ }^{1, b}$, Zhen Chen ${ }^{1, c}$ and Ming Li $i^{1, d}$ \\ ${ }^{1}$ College of Engineering, Ocean University of China, Qingdao, China \\ ameizheng0607@163.com, dimingneu@126.com
}

\begin{abstract}
Keywords: Tidal Stream Turbine, Centralized-control System, Pitch Control, Operation Control Abstract. Marine energy generation systems have gained extensive attention in the past decade. The greatest advantage of tidal current energy over other marine energy sources is its lower dependence on the weather. Tidal stream generation system consists of an energy capturer, a shaft, a gearbox to reach a high rotational speed, a generator, a power converter and submarine power cables for transmission of the generated energy to shore. In such a system, the monitoring and control is very important for the system operation. In this paper, the control system for tidal stream turbines is introduced and the operation control scheme is proposed, the operation control procedure includes system start up, stop, power control, protection etc. The system provides valuable data and experience to further investigate and utilize tidal current energy.
\end{abstract}

\section{Introduction}

The research of tidal stream generation has made great progress in the recent decade [1-3]. In 2003 and 2008, the 300kW SeaFlow and 1.2MW SeaGenS series tidal stream turbines (TST) had been developed by MCT company. The Altantis Resource company of Singapore had developed 1MW AR and AK series TST in 2010 and 2011. In 2013, the 300kW HS300 TST had been developed by Hammerfest Storm company of Norway. In 2013, the 50kW HaiyuanI and HaiyuanII series TST had been developed by Ocean University of China (OUC). And Harbin Engineering University (HEU) had developed $2 * 100 \mathrm{~kW}$ HainengII floating TST in the same year. All these research productions have been studied in many aspects of TST, such as fixing methods, blade design [4,5]and sealing. And they have the ability to operate in real ocean condition.

In order to achieve long-term, large-scale and commercial operation of the TST, many problems need to be studied, such as bad ocean weather, sea water corrosion, adhesion of sea creatures and sealing. The TST will be launched in the ocean and it is an unmanned operation system. It is very necessary to design a monitoring and control system with the functions of monitoring, diagnosis, optimized control.

This paper introduces the composition of monitoring and control system which has been applied in $20 \mathrm{~kW} / 50 \mathrm{~kW} / 100 \mathrm{~kW}$ TSTs developed by OUC. And the operation control scheme is analyzed in detail. The experiment results show that the operation control scheme is validity.

\section{System Composition}

The monitoring and control system of TST presented in this paper is mainly integration of CCS, underwater monitoring system (UMS) and power electronic load (PEL), supplemented by sea conditions information collection and communication system, as shown in Fig.1.

(1) Main functions of UMS consist of two parts, one is monitoring generator and nacelle operation data, such as humidity, temperature, generator speed, output voltage, current and power etc. The other is controlling pitch control system (PCS) adjusting pitch angle. It can communicate with the CCS by CAN bus.

(2) CCS is the control and communication center of the TST. The tidal current information and the UMS information are gathered to CCS. The system operation control is based on the information. The fault diagnosis is also an important function of CCS.

(3) The PEL works in constant power, constant voltage and constant resistant mode. It is controlled by CCS. In the stage of system test, PEL can be controlled adjusting the load precisely for 
tracking maximum output power and test the performance of turbines. When the TST is connected with power grid, it can be used as dump-load to ensure safe operation [6].



Fig.1. General Structure Diagram (a) and Pictures (b) of TST

\section{System Operation Control}

System operation control refers to the functions of decision, execution, control, diagnosis and protection, which is based on the communication between data acquisition and equipment. System operates based on collecting and analysis of tidal current information and system operation data.

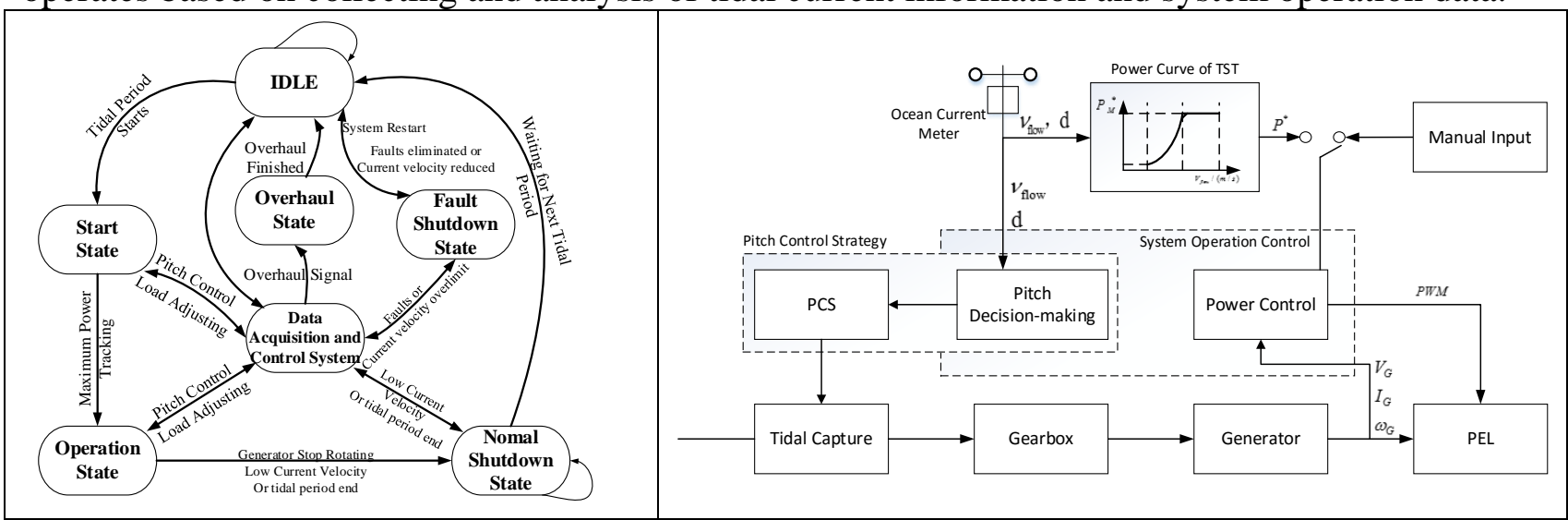

Fig.2 Flow Chart of System Operation State

Fig.3 Operation Control Strategy of TST

According to tidal stream generation characteristics and the design of TST, operation state of the system can be divided into six parts as follows. The current velocity $v_{\text {flow }}$ and current direction $\mathrm{d}$ can be collected by ocean current meter. The system is designed operating under different $v_{\text {flow }}$ and d, which is shown in Fig.2:

1) Idle state:

The PCS and PEL are all in shutdown state waiting for the starting signal. Real-time data monitoring is still in operation to control different state transition under different operation data.

2) Start state:

Tidal current information and generator speed are the main parameters when system starts up. When $v_{\text {flow }}>v_{\text {in }}$, the CCS will control PCS adjusting pitch angle to the best starting angle $\beta_{\text {start }}$ under present $\mathrm{d}$. Then generator can start up under $\beta_{\text {start }}$ more easily. When rotation speed of turbines reaches a threshold, CCS will send a new aiming pitch angle command to PCS, the PCS will adjust pitch angle to $\beta_{\text {opt }}$ gradually, and at the same time, the power of PEL is increasing. The start state finishes and turns into operation state.

3) Operation state:

The CCS adopts different operation control algorithms by collecting $v_{\text {flow }}$. When $v_{\text {in }} \leq v_{\text {flow }} \leq v_{\text {rated }}$, the CCS controls pitch angle maintaining at $\beta_{\mathrm{opt}}$, and generator output power tracking control strategy is proposed. PEL is controlled to adjust load depending on the different $v_{\text {flow }}$. 
When $v_{\text {rated }}<v_{\text {flow }} \leq v_{\text {out }}$, pitch angle at $\beta_{\text {opt }}$ will lead generator speed exceeding rated speed, which will affect the safe operation of TST. The real-time adjusting of PCS according to different $v_{\text {flow }}$ has best effect in theory. But PCS should not operate frequently considering sealing of TST. The segmented operation of PCS can satisfy the basic power control requirement and will reduce the frequency of pitch.

4) Normal shutdown state:

In start state or early operation state, $v_{\text {flow }}$ is generally low. When reducing pitch angle or maintaining $\beta_{\text {opt }}$ can lead to shutdown easily. CCS will control PCS adjusting pitch angle to $\beta_{\text {start }}$, and at the same time reducing the load of PEL. Then turns back to $\beta_{\mathrm{opt}}$ and increases load when monitoring $v_{\text {flow }}$ rising.

According to the characteristics of tidal current and field test data, $v_{\text {flow }}$ decreases significantly at the end of the tidal period. Generator will stop rotating easily at $\beta_{\mathrm{opt}}$. In this case, CCS needs to control the PCS adjusting pitch angle to $\beta_{\text {start }}$, and at the same time reducing the load of PEL until the end of tidal period. Then CCS sends control command to adjust pitch angle to reverse best starting angle $\beta_{\text {start }}^{\prime}$ and switches off the PEL waiting next tidal period.

5) Fault shutdown state:

When $v_{\text {flow }}>v_{\text {out }}$ or other breakdowns, such as underwater turbines fault, load dropping, etc., protection of generator is essential. CCS needs to adjust pitch angle to feathering angle immediately, then controls PEL to switch off until $v_{\text {flow }}$ reduced or faults eliminated.

6) Overhaul state:

When system breakdown or other problems happen that remote control system cannot solve, on-site maintenance needs to be carried out. System turns into overhaul state by remote or local communication. Pitch angle adjusts to feathering angle and PEL switches off. Power supply of maintenance equipment can be controlled to cut off to ensure security of maintenance personnel.

The whole control scheme is shown in Fig.3, which includes the pitch angle control and generator control. According to real-time acquisition of tidal current information, PCS is controlled to adjust pitch angle to achieve maximum captured power under present $v_{\text {flow }}$ and . Then pitch angle will be fixed. During the test stage, the PEL is applied to control the generator power to realize maximum power tracking according to generator power curve [7].

In the preliminary field test, the switch of power control entrance will be turned to manual input. The constant power reference value $P^{*}$ of PEL is manually entered at each $v_{\text {flow }}$. The power curve of the TST can be obtained by connecting the peak phase of each $v_{\text {flow }}$ power curve.

Where $V_{G}, I_{G}$ is output voltage/current of TST, which can be collected by transmitter. $\omega_{G}$ is generator speed, which can be collected by encoder.

\section{Field Test}

The TST had been launched successfully, and operation data had been uploaded by remote communication. Operation state and operation control strategy can be analyzed through those data.

The following Fig.4 shows operation test of generator with PEL. It can be divided into several parts according to system operation procedure, and is analyzed in details as follows:

(1) $7: 10, v_{\text {flow }}$ increases gradually, and is higher than $v_{\text {in }}$. System operation state turns into start state from idle state. And pitch angle changes into best starting angle $\beta_{\text {start }}$. PEL increases the load gradually, and the output power is rising.

(2) $7: 40$, pitch angle is adjusted to about $4^{\circ}\left(\beta_{\text {opt }}\right)$. PEL is adjusted in real-time for maximizing output power according to $v_{\text {flow }}$. System is in operation state and lasts for 3.5 hours.

(3) $11: 10, v_{\text {flow }}$ becomes slow, and generator stops rotating at $\beta_{\text {opt }}$. Pitch angle is controlled to adjusting to $\beta_{\text {start }}$ segmentally. Tidal period is ending, and output power gradually decreases until 
13:00. Then CCS sends control command to adjust pitch angle to reverse best starting angle $\beta_{\text {start }}^{\prime}$ and switches off the PEL waiting next tidal period.

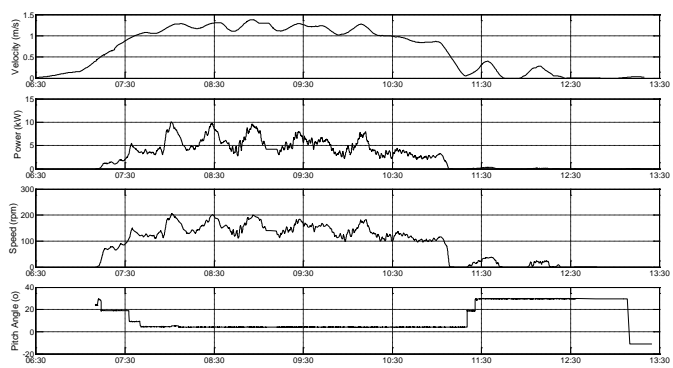

Fig.4. Operation Test of Generator with PEL

\section{Conclusion}

In this paper, the operation control strategy of pitchable horizontal axis generator is systematically introduced. It is based on real-time monitoring of ocean current information and system operation data. The pitch control strategy and generator power control strategy is designed to achieve stable operation, captured power and output power maximizing of TST. Through analysis of system design and those data, the operation control strategy has been proved to ensure stable and reliable operation of the tidal stream turbine.

\section{Acknowledgements}

This work is supported by National Natural Science Foundation (NNSF) of China under Grant 51279185.

\section{References}

[1] J.F. Manwell, J. G. McGowan, and A. L. Rogers. Wind Energy Explained: Theory, Design and Application. Hoboken, NJ, USA: Wiley, 2009.

[2] B. Funieru; A. Binder: Design of a PM direct drive synchronous generator used in a tidal stream turbine. In Clean Electrical Power (ICCEP), Alghero International Conference, Page(s):197-202.

[3] R Vennell, SW Funke, S Draper, C Stevens, T Divett. Designing large arrays of tidal turbines: A synthesis and review. Renewable \& Sustainable Energy Reviews, 2015, 41:454-472.

[4] Z. Zhou; F. Scuiller; J.F. Charpentier; M.E.H. Benbouzid; T. Tang. Power Control of a Nonpitchable PMSG-Based Marine Current Turbine at Overrated Current Speed With Flux-Weakening Strategy. In IEEE Journal of Oceanic Engineering (Volume: 40, Issue: 3), Page(s):536-545.

[5] Chen Zhen; Chi Shukai; Li Ming; Wang Jianguo: Design and Implementation of the Pitch Control System. In Control and Decision Conference (CCDC), 2015 27th Chinese, Page(s):4078-4082 (2015).

[6] Z.Zhou; F.Scuiller; J.F.Charpentier; M.Benbouzid; T.Tang : Grid-Connected Marine Current Generation System Power Smoothing Control Using Supercapacitors. In IECON 2012 38th Annual Conference on IEEE Industrial Electronics Society, Page(s):4035-4040 (2012).

[7] Shenghui Wang ; Ming Li ; Zhen Chen ; Guanghong Chang ; Jianguo Wang ; Shiqi An: Design and implementation of power electronic load used to test tidal current energy generator sets. In: Fuzzy Systems (FUZZ-IEEE), IEEE International Conference (2014). 